Short communication

\title{
Synthesis of novel isoxazolidine derivatives and studies for their antifungal properties
}

\author{
Kodagahally R. Ravi Kumar, Honnaiah Mallesha, Basappa, \\ Kanchugarakoppal S. Rangappa* \\ Department of Studies in Chemistry, University of Mysore, Manasagangotri, Mysore 570 006, India
}

Received 30 September 2002; received in revised form 3 March 2003; accepted 3 March 2003

\begin{abstract}
A series of novel 5-substituted isoxazolidine derivatives $\mathbf{3 a}(\mathbf{i}-\mathbf{v i i i}), \mathbf{3 b}(\mathbf{i}-\mathbf{v i i i})$ and $\mathbf{3 c}(\mathbf{i}-\mathbf{v i i i})$ have been prepared and their antifungal activity on Aspergillus flavus, Fusarium moniliforme and Botrydiplodia theobromae have been evaluated.
\end{abstract}

(C) 2003 Éditions scientifiques et médicales Elsevier SAS. All rights reserved.

Keywords: Antifungal properties; Synthesis; Isoxazolidine derivatives; Cycloaddition

\section{Introduction}

Over the past two decades the frequency of systematic fungal infection in man has increased dramatically. Ketoconazole was the first orally active antifungal agent that was effective against a broad array of systematic and superficial fungal infections [1]. In addition to azole derivatives, a number of antifungal agents having chemical structures other than azoles have recently been introduced to the clinic.

In previous studies [2,3], we have described the application of 1,3-dipolar cycloaddition reaction to the synthesis of biologically active compounds. We recognized an opportunity to apply our knowledge of 1,3dipolar species to the synthesis of a series of 5substituted isoxazolidine compounds through the 1,3dipolar cycloaddition reaction of $\alpha$-substituted aldonitrones and monosubstituted alkenes. The resulting 5substituted isoxazolidines were expected to differ in their chemical and antifungal properties from the known azole derivatives, thus providing valuable information of a new generation of antifungal agents.

* Corresponding author.

E-mail address: rangappaks@yahoo.com (K.S. Rangappa).

\section{Chemistry}

Compounds 2(a-c) were prepared by the reduction of nitro compounds and 4-biphenyl carboxaldehyde with zinc dust using histidine as catalyst as shown in Fig. 1 [4]. The nitrones were found to be light sensitive [5] and hygroscopic [6]. Hence, they were stored in the dark until further use. The nitrones are versatile synthetic intermediates in organic synthesis [7,8]. Recently, we reported that nitrones are convenient class of compounds used for synthesis of ultimate carcinogens [9-11] which are biologically interesting molecules. The novel isoxazolidines $\mathbf{3}(\mathbf{a}-\mathbf{c})$ were obtained by refluxing the nitrones $\mathbf{2}(\mathbf{a}-\mathbf{c})$ with equimolar amount of monosubstituted alkene in toluene-xylene. The cycloaddition of all monosubstituted olefins with nitrone showed high regioselectivity and gave 5-substituted derivatives predominantly major products [12] for both electron donating and electron withdrawing groups.

\section{Results and discussion}

The major products of 5-substituted isoxazolidine derivatives $\mathbf{3}(\mathbf{a}-\mathbf{c})$ were separated on silica gel column using appropriate combination of chloroform, hexane, benzene and petroleum ether as eluent. The reaction condition and the physical data of cycloadducts are 


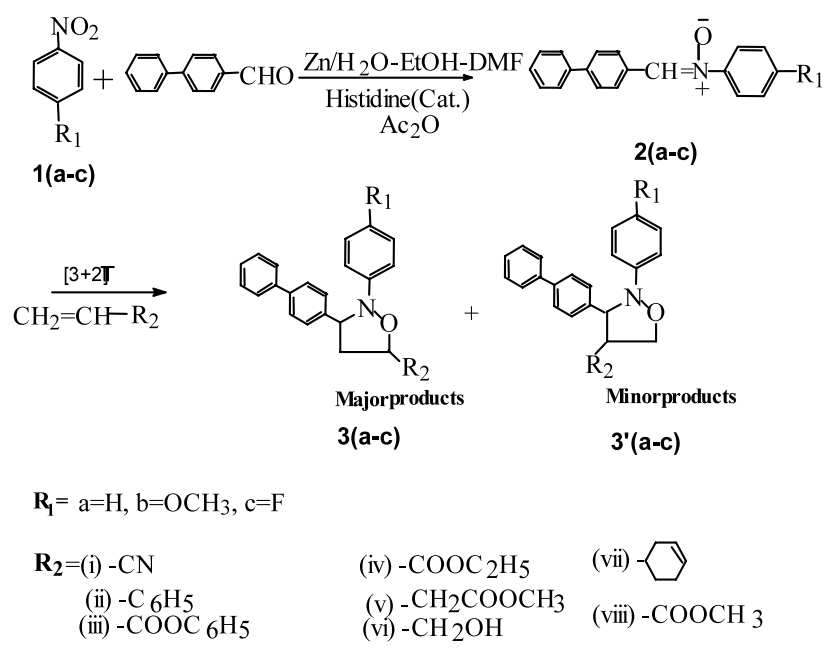

Fig. 1.

given in Table 1. All the synthesized isoxazolidines were structurally characterized by IR, ${ }^{1} \mathrm{H}-\mathrm{NMR}$ and $\mathrm{C}, \mathrm{H}, \mathrm{N}$ analysis. The antifungal activity of these new compounds were evaluated in solid agar media, and their antifungal activity are summarized in Table 2 . Some compounds $\mathbf{3 b}$ (ii), $\mathbf{3 b}$ (iv), $\mathbf{3 b}$ (vi) $\mathbf{3} \mathbf{c}(\mathbf{i i})$, and $\mathbf{3 c}$ (vi) were shown more potent than nystatin. In general, $\mathbf{3 c}(\mathbf{i}-\mathbf{v i i i})$ showed better activity than $\mathbf{3 a}(\mathbf{i}-\mathbf{v i i i})$ and $\mathbf{3 b}(\mathbf{i}-\mathbf{v i i i})$. The introduction of fluorine to the phenyl ring enhanced the antifungal activity. However, introduction of one more phenyl ring to the isoxazolidine ring in $\mathbf{3 b}$ (ii) and $\mathbf{3 c}$ (ii) increasing the antifungal activity and the introduction of methylene hydroxy group in $\mathbf{3 b}(\mathbf{v i})$ and $\mathbf{3 e}(\mathbf{v i})$ of isoxazolidine enhancing the antifungal activity.

\section{Conclusion}

We prepared a novel series of 5-substituted isoxazolidines. Some of these compounds possessed good antifungal activities against the fungus Aspergillus flavus, Fusarium moniliforme and Botrydiplodia theobromae.

\section{Experimental}

\subsection{Chemistry}

The melting points were determined on SELACO-650 hot stage apparatus and are uncorrected. IR (Nujol) spectra were measured on shimadzu 8300 IR spectrophotometer, ${ }^{1} \mathrm{H}-\mathrm{NMR}$ were recorded on Shimadzu AMX 400-Bruker, $400 \mathrm{MHz}$ spectrophotometer by using $\mathrm{CDCl} 3$ as solvent and TMS as an internal standard (chemical shift in $\delta$ ppm). Elemental analyses $(\mathrm{C}, \mathrm{H}, \mathrm{N})$ were obtained on a Vario-EL instrument. The results were within $\pm 0.4 \%$ of the theoretical values. Column and TLC were done with silica gel BDH 60 120 mesh and pre-coated silica gel plates.

Table 1

Reaction condition and physical data of isoxazolidines $\mathbf{3 a}(\mathbf{i}-\mathbf{v i i}), \mathbf{3 b}(\mathbf{i}-\mathbf{v i i})$ and $\mathbf{3 c}(\mathbf{i}$-viii) series

\begin{tabular}{|c|c|c|c|c|c|c|}
\hline Isoxazolidines & Solvent used for reaction & Time taken to complete the reaction (h) & $R_{\mathrm{f}}$ value & Eluent used in separation & $\%$ Yield & M.p. $\left({ }^{\circ} \mathrm{C}\right)$ \\
\hline 3a(i) & toluene & 17 & 0.61 & $\mathrm{CHCl}_{3}+$ hexane $1: 2$ & 57 & oily \\
\hline 3a(ii) & toluene & 17 & 0.63 & $\mathrm{CHCl}_{3}+$ hexane $1: 2$ & 61 & 141 \\
\hline 3a(iii) & toluene & 18 & 0.58 & $\mathrm{CHCl}_{3}+$ petroleum ether $2: 1$ & 54 & oily \\
\hline 3a(iv) & toluene & 18 & 0.54 & $\mathrm{CHCl}_{3}+$ petroleum ether $2: 1$ & 84 & oily \\
\hline $3 a(v)$ & toluene & 31 & 0.68 & $\mathrm{CHCl}_{3}+$ petroleum ether $2: 1$ & 69 & oily \\
\hline 3a(vi) & toluene & 22 & 0.58 & $\mathrm{CHCl}_{3}+$ petroleum ether $2: 1$ & 58.6 & oily \\
\hline $3 a(v i i)$ & toluene & 20 & 0.62 & $\mathrm{CHCl}_{3}+$ petroleum ether $2: 1$ & 62 & oily \\
\hline 3a(viii) & toluene & 18 & 0.64 & $\mathrm{CHCl}_{3}+$ hexane $1: 2$ & 68 & oily \\
\hline $3 b(i)$ & toluene & 19 & 0.62 & $\mathrm{CHCl}_{3}+$ hexane $1: 2$ & 69.5 & oily \\
\hline $3 \mathbf{b}(\mathbf{i i})$ & toluene & 24 & 0.64 & $\mathrm{CHCl}_{3}+$ hexane $1: 2$ & 71.5 & 134 \\
\hline $3 b(\mathrm{iii})$ & toluene & 19 & 0.61 & $\mathrm{CHCl}_{3}+$ hexane $1: 2$ & 65 & oily \\
\hline $3 \mathbf{b}(\mathbf{i v})$ & toluene & 20 & 0.58 & $\mathrm{CHCl}_{3}+$ petroleum ether $1: 1$ & 84 & oily \\
\hline $3 b(v)$ & toluene & 24 & 0.67 & $\mathrm{CHCl}_{3}+$ petroleum ether $2: 1$ & 80 & oily \\
\hline $3 \mathbf{b}(\mathbf{v i})$ & toluene & 17 & 0.69 & $\mathrm{CHCl}_{3}+$ petroleum ether $2: 1$ & 65.5 & oily \\
\hline $3 \mathbf{b}($ vii) & toluene & 22 & 0.62 & $\mathrm{CHCl}_{3}+$ petroleum ether $2: 1$ & 61.5 & oily \\
\hline $3 b($ viii) & toluene & 18 & 0.66 & $\mathrm{CHCl}_{3}+$ petroleum ether $2: 1$ & 64 & oily \\
\hline $3 \mathbf{c}(\mathbf{i})$ & xylene & 20 & 0.77 & $\mathrm{CHCl}_{3}+$ benzene $(2: 1)$ & 65 & oily \\
\hline $3 \mathbf{c}(\mathbf{i i})$ & xylene & 19 & 0.86 & $\mathrm{CHCl}_{3}+$ benzene $(2: 1)$ & 60 & 132 \\
\hline $3 c($ iii) & xylene & 20 & 0.63 & $\mathrm{CHCl}_{3}+$ benzene $(3: 1)$ & 59 & oily \\
\hline $3 c(i v)$ & xylene & 22 & 0.71 & $\mathrm{CHCl}_{3}+$ petroleum ether $(3: 1)$ & 61 & oily \\
\hline $3 \mathbf{c}(\mathbf{v})$ & xylene & 24 & 0.69 & $\mathrm{CHCl}_{3}+$ benzene $(2: 1)$ & 62.8 & oily \\
\hline $3 c(v i)$ & xylene & 24 & 0.68 & $\mathrm{CHCl}_{3}+$ petroleum ether $(2: 1)$ & 59.6 & oily \\
\hline $3 c(v i i)$ & xylene & 23 & 0.79 & $\mathrm{CHCl}_{3}+$ benzene $(1: 1)$ & 66.4 & oily \\
\hline $3 c(v i i i)$ & xylene & 22 & 0.66 & $\mathrm{CHCl}_{3}+$ benzene $(3: 1)$ & 58 & oily \\
\hline
\end{tabular}


Table 2

The minimum inhibitory concentrations $(\mathrm{MIC})^{\mathrm{a}}$ of isoxazolidines $\mathbf{3 a}(\mathbf{i}-\mathbf{v i i i}), \mathbf{3 b}(\mathbf{i}-\mathbf{v i i i})$ and $\mathbf{3 c}(\mathbf{i}-\mathbf{v i i i})$ for antifungal activity

\begin{tabular}{|c|c|c|c|}
\hline \multirow[t]{2}{*}{ Isoxazolidines } & \multicolumn{3}{|l|}{ Tested fungus } \\
\hline & A. flavus (MIC in $\mathrm{mM}$ ) & F. moniliforme (MIC in $\mathrm{mM})$ & B. theobromae ( $\mathrm{MIC}$ in $\mathrm{mM})$ \\
\hline Nystatin & 3 & 5 & 2.5 \\
\hline $3 a(i)$ & 12.0 & 12.0 & 11.5 \\
\hline 3a(ii) & 5.5 & 10.5 & 5.5 \\
\hline 3a(iii) & 7.0 & 5.0 & 9.5 \\
\hline 3a(iv) & 10.5 & 10.5 & 5.5 \\
\hline $3 \mathbf{a}(\mathbf{v})$ & 8.0 & 8.0 & 11.0 \\
\hline $3 a(v i)$ & 12.0 & 3.5 & 12.0 \\
\hline $3 a(v i i)$ & 5.5 & 10.5 & 5.5 \\
\hline 3a(viii) & 11.5 & 5.5 & 11.5 \\
\hline $3 b(\mathbf{i})$ & 8.5 & 8.5 & 7.5 \\
\hline $3 b(i i)$ & 2.5 & 5.0 & 5.0 \\
\hline $3 \mathbf{b}(\mathrm{iii})$ & 4.5 & 6.5 & 7.0 \\
\hline $3 b(i v)$ & 2.5 & 6.0 & 9.0 \\
\hline $3 \mathbf{b}(\mathbf{v})$ & 6.5 & 7.5 & 7.5 \\
\hline $3 b(v i)$ & 3.0 & 3.0 & 5.5 \\
\hline $3 b(v i i)$ & 7.5 & 10.0 & 7.5 \\
\hline $3 b(v i i i)$ & 5.5 & 9.0 & 6.5 \\
\hline $3 c(i)$ & 9.0 & 7.5 & 8.5 \\
\hline $3 \mathbf{c}(\mathbf{i i})$ & 2.5 & 5.5 & 5.0 \\
\hline $3 c($ iii) & 5.5 & 7.0 & 6.0 \\
\hline $3 c(i v)$ & 4.0 & 5.5 & 7.5 \\
\hline $3 c(v)$ & 8.0 & 6.5 & 9.0 \\
\hline $3 c(v i)$ & 3.0 & 3.0 & 6.0 \\
\hline $3 c(v i i)$ & 5.0 & 5.5 & 7.5 \\
\hline $3 c(v i i i)$ & 8.0 & 6.5 & 8.0 \\
\hline
\end{tabular}

a Average of atleast three determinations.

General procedure for the synthesis of isoxazolidines, $\mathbf{3 a}$ (i-viii), $\mathbf{3 b} \mathbf{b}(\mathbf{i}-\mathbf{v i i})$ and $\mathbf{3 c}(\mathbf{i}-\mathbf{v i i i})$ : Equimolar mixture of nitrones $\mathbf{2 a}, \mathbf{2 b}$ and $\mathbf{2 c}$ and different alkenes were dissolved in $10 \mathrm{~mL}$ of toluene-xylene. The reaction mixture was refluxed for $18-20 \mathrm{~h}$ to complete the reaction, which was monitored by TLC. The pure products were separated by using appropriate mixture of chloroform, hexane, petroleum ether and benzene as eluent in silica gel column.

\subsubsection{Synthesis of C-biphenyl- $N$-phenyl nitrone (2a)}

This was obtained from the reduction of a mixture of nitrobenzene and 4-biphenyl carboxaldehyde with zinc dust using histidine as catalyst [4]. A white crystalline solid is obtained in ethanol; yield $748 \mathrm{mg}(80 \%)$, m.p. $203^{\circ} \mathrm{C}$.

${ }^{1} \mathrm{H}-\mathrm{NMR} \delta(\mathrm{ppm}): 7.39-7.51(\mathrm{~m}, 6 \mathrm{H}, \mathrm{Ar}-\mathrm{H}), 7.65-$ $7.48(\mathrm{~m}, 6 \mathrm{H}, \mathrm{Ar}-\mathrm{H}), 7.97(\mathrm{~s}, 1 \mathrm{H}, \mathrm{CH}=\mathrm{N}), 8.46-8.51$ (dd, $2 \mathrm{H}, \mathrm{Ar}-\mathrm{H})$.

IR (Nujol): $v\left(\mathrm{~cm}^{-1}\right): 1584(\mathrm{C}=\mathrm{N}) ; 1168(\mathrm{NO})$.

\subsubsection{Synthesis of C-biphenyl-N-(4- methoxyphenyl)nitrone ( $2 \boldsymbol{b}$ )}

This was obtained from the reduction of a mixture of 4-methoxynitrobenzene and 4-biphenyl carboxaldehyde with zinc dust using histidine as catalyst. A white crystalline solid is obtained in ethanol; yield $815 \mathrm{mg}$ (95\%), m.p. $178^{\circ} \mathrm{C}$.

${ }^{1} \mathrm{H}-\mathrm{NMR} \delta$ (ppm): 3.82 (s, 3H, $\left.\mathrm{Ar}-\mathrm{OCH}_{3}\right) ; 7.20-7.24$ $(\mathrm{d}, 2 \mathrm{H}, \mathrm{Ar}-\mathrm{H}) ; 7.34-7.46(\mathrm{~m}, 3 \mathrm{H}, \mathrm{Ar}-\mathrm{H}), 7.62-7.72$ $(\mathrm{m}, 6 \mathrm{H}, \mathrm{Ar}-\mathrm{H}), 7.92(\mathrm{~s}, 1 \mathrm{H}, \mathrm{CH}=\mathrm{N}), 8.24-8.28(\mathrm{~d}, 2 \mathrm{H}$, $\mathrm{Ar}-\mathrm{H})$.

IR (Nujol): $v\left(\mathrm{~cm}^{-1}\right): 1572(\mathrm{C}=\mathrm{N}) ; 1152(\mathrm{NO})$.

\subsubsection{Synthesis of C-biphenyl- $N-(4-$}

fluorophenyl)nitrone (2c)

This was obtained from the reduction of a mixture of 4-fluoronitrobenzene and 4-biphenyl carboxaldehyde with zinc dust using histidine as catalyst [4]. A white crystalline solid is obtained in ethanol; yield $812 \mathrm{mg}$ (96\%), m.p. $307^{\circ} \mathrm{C}$.

${ }^{1} \mathrm{H}-\mathrm{NMR} \delta$ (ppm): 7.44-7.49 (m, 5H, Ar-H), 7.65$7.79(\mathrm{~m}, 6 \mathrm{H}, \mathrm{Ar}-\mathrm{H}), 7.94(\mathrm{~s}, 1 \mathrm{H}, \mathrm{CH}=\mathrm{N}), 8.44-8.48(\mathrm{~d}$, $2 \mathrm{H}, \mathrm{Ar}-\mathrm{H})$.

IR (Nujol): $v\left(\mathrm{~cm}^{-1}\right): 1572(\mathrm{C}=\mathrm{N}) ; 1152(\mathrm{NO})$.

\subsubsection{Synthesis of 2-phenyl-3-biphenyl-5-cyano isoxazolidine $(\mathbf{3 a}(\boldsymbol{i}))$}

This was obtained from equimolar mixture of $C$-(4biphenyl)- $N$-phenylnitrone (2a) $(500 \mathrm{mg})$ and acrylonitrile $(0.10 \mathrm{~mL})$.

${ }^{1} \mathrm{H}-\mathrm{NMR} \delta(\mathrm{ppm}): 2.65-2.81\left(\mathrm{dd}, 2 \mathrm{H}, \mathrm{H}_{4}\right) ; 4.45(\mathrm{t}$, $\left.1 \mathrm{H}, \mathrm{H}_{5}\right) ; 5.08\left(\mathrm{t}, 1 \mathrm{H}, \mathrm{H}_{3}\right) ; 6.91(\mathrm{t}, 1 \mathrm{H}, \mathrm{Ar}-\mathrm{H}) ; 7.2(\mathrm{t}, 2 \mathrm{H}$, 
$\mathrm{Ar}-\mathrm{H}) ; 7.41(\mathrm{~d}, 2 \mathrm{H}, \mathrm{Ar}-\mathrm{H}(\mathrm{BP}) ; 7.52(\mathrm{t}, 1 \mathrm{H}, \mathrm{Ar}-\mathrm{H}(\mathrm{BP})$; 7.64 (t, 2H, Ar-H(BP); $7.72(\mathrm{~d}, 2 \mathrm{H}, \mathrm{Ar}-\mathrm{H}) ; 7.69$ (d, 4H, $\mathrm{Ar}-\mathrm{H}(\mathrm{BP})$.

IR (Nujol): $v\left(\mathrm{~cm}^{-1}\right): 1742$ (CO); 1280 (NO).

\subsubsection{Synthesis of 2-phenyl-3-biphenyl-5-phenyl isoxazolidine ( $\mathbf{3 a}(\boldsymbol{i i})$ )}

This was obtained from equimolar mixture of $C$-(4biphenyl)- $N$-phenylnitrone (2a) $(500 \mathrm{mg})$ and styrene $(0.25 \mathrm{~mL})$.

${ }^{1} \mathrm{H}-\mathrm{NMR} \delta(\mathrm{ppm}): 2.72-2.88\left(\mathrm{dd}, 2 \mathrm{H}, \mathrm{H}_{4}\right) ; 5.05(\mathrm{t}$, $\left.1 \mathrm{H}, \mathrm{H}_{5}\right) ; 5.12\left(\mathrm{t}, 1 \mathrm{H}, \mathrm{H}_{3}\right) ; 6.91(\mathrm{t}, 1 \mathrm{H}, \mathrm{Ar}-\mathrm{H}) ; 7.2(\mathrm{t}, 2 \mathrm{H}$, $\mathrm{Ar}-\mathrm{H}) ; 7.29-7.41(\mathrm{~m}, 5 \mathrm{H}, \mathrm{Ar}-\mathrm{H}) ; 7.42(\mathrm{~d}, 2 \mathrm{H}, \mathrm{Ar}-$ $\mathrm{H}(\mathrm{BP}) ; 7.52(\mathrm{t}, 1 \mathrm{H}, \mathrm{Ar}-\mathrm{H}(\mathrm{BP}) ; 7.64(\mathrm{t}, 2 \mathrm{H}, \mathrm{Ar}-$ $\mathrm{H}(\mathrm{BP}) ; 7.72$ (d, 2H, Ar-H); 7.69 (d, 4H, Ar-H(BP).

IR (Nujol): $v\left(\mathrm{~cm}^{-1}\right)$ : 1712 (CO); 1240 (NO).

\subsubsection{Synthesis of 2-phenyl-3-biphenyl-5-benzoate isoxazolidine ( $\mathbf{3 a}(\boldsymbol{i i i})$ )}

This was obtained from equimolar mixture of $C$-(4biphenyl)- $N$-phenylnitrone (2a) $(500 \mathrm{mg})$ and vinyl benzoate $(0.25 \mathrm{~mL})$.

${ }^{1} \mathrm{H}-\mathrm{NMR} \delta(\mathrm{ppm}): 2.70-2.86\left(\mathrm{dd}, 2 \mathrm{H}, \mathrm{H}_{4}\right) ; 4.72(\mathrm{t}$, $\left.1 \mathrm{H}, \mathrm{H}_{5}\right) ; 5.13\left(\mathrm{t}, 1 \mathrm{H}, \mathrm{H}_{3}\right) ; 6.91(\mathrm{t}, 1 \mathrm{H}, \mathrm{Ar}-\mathrm{H}) ; 7.20(\mathrm{t}$, $2 \mathrm{H}, \mathrm{Ar}-\mathrm{H}) ; 7.28-7.50(\mathrm{~m}, 3 \mathrm{H}, \mathrm{Ar}-\mathrm{H}) ; 7.41(\mathrm{~d}, 2 \mathrm{H}, \mathrm{Ar}-$ $\mathrm{H}(\mathrm{BP}) ; 7.52$ (t, 1H, Ar-H(BP); 7.72 (d, 2H, Ar-H); 7.64 (t, 2H, Ar-H(BP); 7.79 (d, 4H, Ar-H(BP); 7.81 (d, 2H, $\mathrm{Ar}-\mathrm{H})$.

IR (Nujol): $v\left(\mathrm{~cm}^{-1}\right): 1748(\mathrm{C}=\mathrm{O}) ; 1730(\mathrm{CO}) ; 1260$ (NO).

\subsubsection{Synthesis of 2-phenyl-3-biphenyl-5-ethylate isoxazolidine ( $3 \boldsymbol{a}(\boldsymbol{i v}))$}

This was obtained from equimolar mixture of $C$-(4biphenyl)- $N$-phenylnitrone (2a) $(500 \mathrm{mg}$ ) and ethyl acrylate $(0.20 \mathrm{~mL})$.

${ }^{1} \mathrm{H}-\mathrm{NMR} \delta(\mathrm{ppm}): 0.94\left(\mathrm{t}, 3 \mathrm{H}, \mathrm{CH}_{3}\right) ; 2.68-2.86(\mathrm{dd}$, $\left.2 \mathrm{H}, \mathrm{H}_{4}\right) ; 3.62\left(\mathrm{q}, 2 \mathrm{H}, \mathrm{CH}_{2}\right) ; 4.43\left(\mathrm{t}, 1 \mathrm{H}, \mathrm{H}_{5}\right) ; 5.12(\mathrm{t}, 1 \mathrm{H}$, $\left.\mathrm{H}_{3}\right) ; 6.91(\mathrm{t}, 1 \mathrm{H}, \mathrm{Ar}-\mathrm{H}) ; 7.2(\mathrm{t}, 2 \mathrm{H}, \mathrm{Ar}-\mathrm{H}) ; 7.41(\mathrm{~d}, 2 \mathrm{H}$, $\mathrm{Ar}-\mathrm{H}, \mathrm{BP}) ; 7.52$ (t, 1H, Ar-H, BP); 7.64 (t, 2H, Ar-H, $\mathrm{BP}) ; 7.72$ (d, 2H, Ar-H); 7.79 (d, 4H, Ar-H, BP).

IR (Nujol): $v\left(\mathrm{~cm}^{-1}\right): 1720(\mathrm{C}=\mathrm{O}) ; 1722(\mathrm{CO}) ; 1234$ (NO).

\subsubsection{Synthesis of 2-phenyl-3-biphenyl-5-methylene acetate isoxazolidine $(\mathbf{3 a}(\boldsymbol{v}))$}

This was obtained from equimolar mixture of $C$-(4biphenyl)- $N$-phenylnitrone (2a) $(500 \mathrm{mg})$ and allyl acetate $(0.2 \mathrm{~mL})$.

${ }^{1} \mathrm{H}-\mathrm{NMR} \delta(\mathrm{ppm}): 2.42\left(\mathrm{~d}, 2 \mathrm{H}, \mathrm{CH}_{2}\right) 2.62-2.78(\mathrm{dd}$, $\left.2 \mathrm{H}, \mathrm{H}_{4}\right) ; 3.42\left(\mathrm{~s}, 3 \mathrm{H}, \mathrm{OCH}_{3}\right) ; 2.70-4.25\left(\mathrm{~m}, 1 \mathrm{H}, \mathrm{H}_{5}\right)$; $5.06\left(\mathrm{t}, 1 \mathrm{H}, \mathrm{H}_{3}\right) 6.91(\mathrm{t}, 1 \mathrm{H}, \mathrm{Ar}-\mathrm{H}) ; 7.2(\mathrm{t}, 2 \mathrm{H}, \mathrm{Ar}-\mathrm{H})$; $7.41(\mathrm{~d}, 2 \mathrm{H}, \mathrm{Ar}-\mathrm{H}, \mathrm{BP}) ; 7.52(\mathrm{t}, 1 \mathrm{H}, \mathrm{Ar}-\mathrm{H}, \mathrm{BP}) ; 7.64(\mathrm{t}$, 2H, Ar-H, BP); 7.72 (d, 2H, Ar-H); 7.69 (d, 4H, Ar-H, $\mathrm{BP})$.

IR (Nujol): $v\left(\mathrm{~cm}^{-1}\right)$ : 1745 (CO); 1270 (NO).
5.1.9. Synthesis of 2-phenyl-3-biphenyl-5-methelene hydroxy isoxazolidine $(\mathbf{3 a}(\boldsymbol{v i}))$

This was obtained from equimolar mixture of $C$-(4biphenyl)- $N$-phenylnitrone (2a) $(500 \mathrm{mg})$ and allyl alcohol $(0.12 \mathrm{~mL})$.

${ }^{1} \mathrm{H}-\mathrm{NMR} \delta(\mathrm{ppm}): 2.65-2.80\left(\mathrm{dd}, 2 \mathrm{H}, \mathrm{H}_{4}\right) ; 3.64(\mathrm{~d}$, $\left.2 \mathrm{H}, \mathrm{CH}_{2}\right) ; 4.76\left(\mathrm{~m}, 1 \mathrm{H}, \mathrm{H}_{5}\right) ; 5.14\left(\mathrm{t}, 1 \mathrm{H}, \mathrm{H}_{3}\right) ; 5.25(\mathrm{~s}$, $1 \mathrm{H}, \mathrm{OH}) ; 6.92(\mathrm{t}, 1 \mathrm{H}, \mathrm{Ar}-\mathrm{H}) ; 7.2(\mathrm{t}, 2 \mathrm{H}, \mathrm{Ar}-\mathrm{H}) ; 7.41(\mathrm{~d}$, $2 \mathrm{H}, \mathrm{Ar}-\mathrm{H}, \mathrm{BP}) ; 7.52(\mathrm{t}, 1 \mathrm{H}, \mathrm{Ar}-\mathrm{H}, \mathrm{BP}) ; 7.64$ (t, 2H, $\mathrm{Ar}-\mathrm{H}, \mathrm{BP}) ; 7.72$ (d, 2H, Ar-H); 7.69 (d, 4H, Ar-H, $\mathrm{BP})$.

IR (Nujol): $v\left(\mathrm{~cm}^{-1}\right)$ : 1710 (CO); 1242 (NO).

\subsubsection{Synthesis of 2-phenyl-3-biphenyl-5-(3-} cyclohexene) isoxazolidine (3a(vii))

This was obtained from equimolar mixture of $C$-(4biphenyl)- $N$-phenylnitrone (3a) (500 mg) and 4-vinyl-1cyclohexene $(0.25 \mathrm{~mL})$.

${ }^{1} \mathrm{H}-\mathrm{NMR} \delta$ (ppm): $1.38-1.42\left(\mathrm{~m}, 2 \mathrm{H}, \mathrm{CH}_{2}\right.$, cyclohexene); $1.52(\mathrm{~s}, 1 \mathrm{H}$, cyclohexene); 2.27-2.31 (m, 2H, cyclohexene); 2.33-2.38 (m, 2H, cyclohexene); 2.62.65-2.80 (dd, $\left.2 \mathrm{H}, \mathrm{H}_{4}\right) ; 3.64\left(\mathrm{~d}, 2 \mathrm{H}, \mathrm{CH}_{2}\right) ; 4.76(\mathrm{t}$, $\left.1 \mathrm{H}, \mathrm{H}_{5}\right) ; 5.14\left(\mathrm{t}, 1 \mathrm{H}, \mathrm{H}_{3}\right) ; 4.82(\mathrm{~m}, 1 \mathrm{H}, \mathrm{CH}) ; 5.12(\mathrm{t}, 1 \mathrm{H}$, $\mathrm{CH})$; 5.6-5.66 (m, 2H, cyclohexene); $6.92(\mathrm{t}, 1 \mathrm{H}, \mathrm{Ar}-$ $\mathrm{H}) ; 7.2(\mathrm{t}, 2 \mathrm{H}, \mathrm{Ar}-\mathrm{H}) ; 7.41(\mathrm{~d}, 2 \mathrm{H}, \mathrm{Ar}-\mathrm{H}, \mathrm{BP}) ; 7.52(\mathrm{t}$, $1 \mathrm{H}, \mathrm{Ar}-\mathrm{H}, \mathrm{BP}) ; 7.64(\mathrm{t}, 2 \mathrm{H}, \mathrm{Ar}-\mathrm{H}, \mathrm{BP}) ; 7.72(\mathrm{~d}, 2 \mathrm{H}$, $\mathrm{Ar}-\mathrm{H}) ; 7.79$ (d, 4H, Ar-H, BP).

IR (Nujol): $v\left(\mathrm{~cm}^{-1}\right)$ : 1730 (CO); $1262(\mathrm{NO})$.

\subsubsection{Synthesis of 2-phenyl-3-biphenyl-5-methylate isoxazolidine (3a(viii))}

This was obtained from equimolar mixture of $C$-(4biphenyl)- $N$-phenylnitrone (3a) (500 mg) and methyl acrylate $(0.17 \mathrm{~mL})$

${ }^{1} \mathrm{H}-\mathrm{NMR} \delta(\mathrm{ppm}): 2.6-2.9\left(\mathrm{dd}, 2 \mathrm{H}, \mathrm{CH}_{2}\right) ; 3.41(\mathrm{~s}$, $\left.3 \mathrm{H}, \mathrm{OCH}_{3}\right) ; 4.45(\mathrm{t}, 1 \mathrm{H}, \mathrm{CH}) ; 5.12(\mathrm{t}, 1 \mathrm{H}, \mathrm{CH}) ; 6.92(\mathrm{t}$, $1 \mathrm{H}, \mathrm{Ar}-\mathrm{H}) ; 7.2(\mathrm{t}, 2 \mathrm{H}, \mathrm{Ar}-\mathrm{H}) ; 7.41(\mathrm{~d}, 2 \mathrm{H}, \mathrm{Ar}-\mathrm{H}, \mathrm{BP})$; $7.52(\mathrm{t}, 1 \mathrm{H}, \mathrm{Ar}-\mathrm{H}, \mathrm{BP}) ; 7.64(\mathrm{t}, 2 \mathrm{H}, \mathrm{Ar}-\mathrm{H}, \mathrm{BP}) ; 7.72$ (d, $2 \mathrm{H}, \mathrm{Ar}-\mathrm{H}) ; 7.79$ (d, 4H, Ar-H, BP).

IR (Nujol): $v\left(\mathrm{~cm}^{-1}\right)$ : 1742 (CO); 1280 (NO).

\subsubsection{Synthesis of 2-(4-methoxyphenyl)-3-biphenyl-5-} cyano isoxazolidine $(\mathbf{3} \boldsymbol{b}(\boldsymbol{i}))$

This was obtained from equimolar mixture of $C$-(4biphenyl)- $N$-(4-methoxyphenyl)nitrone (2b) $(500 \mathrm{mg})$ and acrylonitrile $(0.31 \mathrm{~mL})$.

${ }^{1} \mathrm{H}-\mathrm{NMR} \delta(\mathrm{ppm}): 2.82-3.0\left(\mathrm{dd}, 2 \mathrm{H}, \mathrm{H}_{4}\right) ; 3.76(\mathrm{~s}, 3 \mathrm{H}$, $\left.\mathrm{Ar}-\mathrm{OCH}_{3}\right) ; 4.44\left(\mathrm{t}, 1 \mathrm{H}, \mathrm{H}_{5}\right) ; 5.06\left(\mathrm{t}, 1 \mathrm{H}, \mathrm{H}_{3}\right) ; 7.28(\mathrm{~d}$, $2 \mathrm{H}, \mathrm{Ar}-\mathrm{H}) ; 7.42(\mathrm{~d}, 2 \mathrm{H}, \mathrm{Ar}-\mathrm{H}(\mathrm{BP}) ; 7.52(\mathrm{t}, 1 \mathrm{H}, \mathrm{Ar}-$ $\mathrm{H}(\mathrm{BP}) ; 7.63$ (t, 2H, Ar-H(BP); 7.68 (d, 2H, Ar-H); 7.74 (d, 4H, Ar-H(BP).

IR (Nujol): $v\left(\mathrm{~cm}^{-1}\right)$ : 1748 (CO); 1278 (NO). 
5.1.13. Synthesis of 2-(4-methoxyphenyl)-3-biphenyl-5phenyl isoxazolidine (3b $(\boldsymbol{i i}))$

This was obtained from equimolar mixture of $C$-(4biphenyl- $N$-(4-methoxyphenyl)nitrone (2b) $(500 \mathrm{mg})$ and styrene $(0.25 \mathrm{~mL})$.

${ }^{1} \mathrm{H}-\mathrm{NMR} \delta(\mathrm{ppm}): 2.74-2.92\left(\mathrm{dd}, 2 \mathrm{H}, \mathrm{H}_{4}\right) ; 3.80(\mathrm{~s}$, $\left.3 \mathrm{H}, \mathrm{Ar}-\mathrm{OCH}_{3}\right) ; 5.04\left(\mathrm{t}, 1 \mathrm{H}, \mathrm{H}_{5}\right) ; 5.13\left(\mathrm{t}, 1 \mathrm{H}, \mathrm{H}_{3}\right) ; 7.28-$ $7.40(\mathrm{~m}, 5 \mathrm{H}, \mathrm{Ar}-\mathrm{H}) ; 7.28(\mathrm{~d}, 2 \mathrm{H}, \mathrm{Ar}-\mathrm{H}) ; 7.68(\mathrm{~d}, 2 \mathrm{H}$, $\mathrm{Ar}-\mathrm{H}) ; 7.42$ (d, 2H, Ar-H(BP); 7.54 (t, 1H, Ar-H(BP); 7.64 (t, 2H, Ar-H(BP); 7.72 (d, 4H, Ar-H(BP).

IR (Nujol): $v\left(\mathrm{~cm}^{-1}\right): 1712$ (CO); 1246 (NO).

\subsubsection{Synthesis of 2-(4-methoxyphenyl)-3-biphenyl-5-} benzoate isoxazolidine ( $\mathbf{3 b}(\mathbf{i i i}))$

This was obtained from equimolar mixture of $C$-(4biphenyl)- $N$-(4-methoxyphenyl)nitrone (2b) (500 mg) and vinyl benzoate $(0.26 \mathrm{~mL})$.

${ }^{1} \mathrm{H}-\mathrm{NMR} \delta(\mathrm{ppm}): 2.74-2.92\left(\mathrm{dd}, 2 \mathrm{H}, \mathrm{H}_{4}\right) ; 3.78(\mathrm{~s}$, $\left.3 \mathrm{H}, \mathrm{Ar}-\mathrm{OCH}_{3}\right) ; 4.74\left(\mathrm{t}, 1 \mathrm{H}, \mathrm{H}_{5}\right) ; 5.14\left(\mathrm{t}, 1 \mathrm{H}, \mathrm{H}_{3}\right) ; 7.26-$ $7.50(\mathrm{~m}, 3 \mathrm{H}, \mathrm{Ar}-\mathrm{H}) ; 7.28(\mathrm{~d}, 2 \mathrm{H}, \mathrm{Ar}-\mathrm{H}) ; 7.63(\mathrm{t}, 2 \mathrm{H}$, $\mathrm{Ar}-\mathrm{H}(\mathrm{BP}) 7.70$ (d, 2H, Ar-H); 7.42 (d, 2H, Ar-H(BP); 7.52 (t, 1H, Ar-H(BP); 7.68 (d, 2H, Ar-H); 7.74 (d, 4H, $\mathrm{Ar}-\mathrm{H}(\mathrm{BP})$.

IR (Nujol): $v\left(\mathrm{~cm}^{-1}\right): 1752(\mathrm{C}=\mathrm{O}) ; 1732(\mathrm{CO}) ; 1270$ (NO).

\subsubsection{Synthesis of 2-(4-methoxyphenyl)-3-biphenyl-5-} ethylate isoxazolidine ( $\mathbf{3} \boldsymbol{b}(\boldsymbol{i v})$ )

This was obtained from equimolar mixture of $C$-(4biphenyl- $N$-(4-methoxyphenyl)nitrone (2b) $(500 \mathrm{mg})$ and ethyl acrylate $(0.17 \mathrm{~mL})$.

${ }^{1} \mathrm{H}-\mathrm{NMR} \delta(\mathrm{ppm}): 0.94\left(\mathrm{t}, 3 \mathrm{H}, \mathrm{CH}_{3}\right) ; 2.68-2.85(\mathrm{dd}$, $\left.2 \mathrm{H}, \mathrm{H}_{4}\right) ; 3.62\left(\mathrm{q}, 2 \mathrm{H}, \mathrm{CH}_{2}\right) ; 3.78\left(\mathrm{~s}, 3 \mathrm{H}, \mathrm{Ar}-\mathrm{OCH}_{3}\right) ;$ $4.45\left(\mathrm{t}, 1 \mathrm{H}, \mathrm{H}_{5}\right) ; 5.13\left(\mathrm{t}, 1 \mathrm{H}, \mathrm{H}_{3}\right) ; 7.28(\mathrm{~d}, 2 \mathrm{H}, \mathrm{Ar}-\mathrm{H})$; $7.42(\mathrm{~d}, 2 \mathrm{H}, \mathrm{Ar}-\mathrm{H}(\mathrm{BP}) ; 7.52(\mathrm{t}, 1 \mathrm{H}, \mathrm{Ar}-\mathrm{H}(\mathrm{BP}) ; 7.63(\mathrm{t}$, $2 \mathrm{H}, \mathrm{Ar}-\mathrm{H}(\mathrm{BP}) ; 7.68(\mathrm{~d}, 2 \mathrm{H}, \mathrm{Ar}-\mathrm{H}) ; 7.74(\mathrm{~d}, 4 \mathrm{H}, \mathrm{Ar}-$ $\mathrm{H}(\mathrm{BP})$.

IR (Nujol): $v\left(\mathrm{~cm}^{-1}\right): 1726(\mathrm{C}=\mathrm{O}) ; 1708(\mathrm{CO}) ; 1240$ (NO).

5.1.16. Synthesis of 2-(4-methoxyphenyl)-3-biphenyl-5methylene acetate isoxazolidine $(\boldsymbol{3} \boldsymbol{b}(\boldsymbol{v}))$

This was obtained from equimolar mixture of $C$-(4biphenyl)- $N$-(4-methoxyphenyl)nitrone (2b) $(500 \mathrm{mg})$ and allyl acetate $(0.23 \mathrm{~mL})$.

${ }^{1} \mathrm{H}-\mathrm{NMR} \delta$ (ppm): $2.42\left(\mathrm{~d}, 2 \mathrm{H}, \mathrm{CH}_{2}\right) ; 2.67-2.85(\mathrm{dd}$, $\left.2 \mathrm{H}, \mathrm{H}_{4}\right) ; 3.42\left(\mathrm{~s}, 3 \mathrm{H}, \mathrm{OCH}_{3}\right) ; 3.76\left(\mathrm{~s}, 3 \mathrm{H}, \mathrm{Ar}-\mathrm{OCH}_{3}\right)$; $4.26\left(\mathrm{t}, 1 \mathrm{H}, \mathrm{H}_{5}\right) ; 5.12\left(\mathrm{t}, 1 \mathrm{H}, \mathrm{H}_{3}\right) ; 7.26(\mathrm{~d}, 2 \mathrm{H}, \mathrm{Ar}-\mathrm{H})$; $7.42(\mathrm{~d}, 2 \mathrm{H}, \mathrm{Ar}-\mathrm{H}(\mathrm{BP}) ; 7.50(\mathrm{t}, 1 \mathrm{H}, \mathrm{Ar}-\mathrm{H}(\mathrm{BP}) ; 7.62(\mathrm{t}$, $2 \mathrm{H}, \mathrm{Ar}-\mathrm{H}(\mathrm{BP}) ; 7.68$ (d, 2H, $\mathrm{Ar}-\mathrm{H}) ; 7.70$ (d, 4H, Ar$\mathrm{H}(\mathrm{BP})$.

IR (Nujol): $v\left(\mathrm{~cm}^{-1}\right): 1720(\mathrm{CO}) ; 1250$ (NO).
5.1.17. Synthesis of 2-(4-methoxyphenyl)-3-biphenyl5 metylene hydroxy isoxazolidine ( $\mathbf{3} \boldsymbol{b}(\boldsymbol{v i}))$

This was obtained from equimolar mixture of $C$-(4biphenyl)- $N$-(4-methoxyphenyl)nitrone (2b) (500 mg) and allyl alcohol $(0.24 \mathrm{~mL})$.

${ }^{1} \mathrm{H}-\mathrm{NMR} \delta(\mathrm{ppm}): 2.66-2.84\left(\mathrm{dd}, 2 \mathrm{H}, \mathrm{H}_{4}\right) ; 3.63(\mathrm{~d}$, $\left.2 \mathrm{H}, \mathrm{CH}_{2}\right) ; 3.78\left(\mathrm{~s}, 3 \mathrm{H}, \mathrm{Ar}-\mathrm{OCH}_{3}\right) ; 4.74\left(\mathrm{t}, 1 \mathrm{H}, \mathrm{H}_{5}\right) ; 5.12$ $\left(\mathrm{t}, 1 \mathrm{H}, \mathrm{H}_{3}\right) ; 5.25(\mathrm{~s}, 1 \mathrm{H}, \mathrm{OH}) ; 7.26(\mathrm{~d}, 2 \mathrm{H}, \mathrm{Ar}-\mathrm{H}) ; 7.42$ (d, 2H, Ar-H(BP); 7.52 (t, 1H, Ar-H(BP); 7.63 (t, 2H, $\mathrm{Ar}-\mathrm{H}(\mathrm{BP}) ; 7.68$ (d, 2H, $\mathrm{Ar}-\mathrm{H}) ; 7.74$ (d, 4H, Ar-H(BP).

IR (Nujol): $v\left(\mathrm{~cm}^{-1}\right)$ : 1728 (CO); 1262 (NO).

5.1.18. Synthesis of 2-(4-methoxyphenyl)-3-biphenyl-5(3-cyclohexene) isoxazolidine (3b(vii))

This was obtained from equimolar mixture of $C$-(4biphenyl)- $N$-(4-methoxyphenyl)nitrone (2b) $(500 \mathrm{mg}$ ) and 4-vinyl-1-cyclohexene $(0.21 \mathrm{~mL})$.

${ }^{1} \mathrm{H}-\mathrm{NMR} \delta$ (ppm): 1.37-1.41 (m, 2H, $\mathrm{CH}_{2}$, cyclohexene); $1.52(\mathrm{~s}, 1 \mathrm{H}$, cyclohexene); $2.26-2.30(\mathrm{~m}, 2 \mathrm{H}$, cyclohexene); 2.32-2.36 (m, 2H, cyclohexene); 2.68$2.86\left(\mathrm{dd}, 2 \mathrm{H}, \mathrm{H}_{4}\right) ; 3.76\left(\mathrm{~s}, 3 \mathrm{H}, \mathrm{Ar}-\mathrm{OCH}_{3}\right) 4.82(\mathrm{~m}$, $\left.1 \mathrm{H}, \mathrm{H}_{5}\right) ; 5.12\left(\mathrm{t}, 1 \mathrm{H}, \mathrm{H}_{3}\right) ; 5.60-5.68(\mathrm{~m}, 2 \mathrm{H}$, cyclohexene); 7.28 (d, 2H, $\mathrm{Ar}-\mathrm{H}) ; 7.42(\mathrm{~d}, 2 \mathrm{H}, \mathrm{Ar}-\mathrm{H}, \mathrm{BP}) ; 7.52$ (t, 1H, Ar-H, BP); 7.63 (t, 2H, Ar-H, BP); 7.68 (d, 2H, $\mathrm{Ar}-\mathrm{H}) ; 7.79$ (d, 4H, Ar-H, BP).

IR (Nujol): $v\left(\mathrm{~cm}^{-1}\right)$ :1744 (CO); 1270 (NO).

5.1.19. Synthesis of 2-(4-methoxyphenyl)-3-biphenyl-5methylate isoxazolidine (3b(viii))

This was obtained from equimolar mixture of $C$-(4biphenyl- $N$-(4-methoxyphenyl)nitrone (2b) $(500 \mathrm{mg})$ and methyl acrylate $(0.17 \mathrm{~mL})$.

${ }^{1} \mathrm{H}-\mathrm{NMR} \delta(\mathrm{ppm}): 2.66-2.84\left(\mathrm{dd}, 2 \mathrm{H}, \mathrm{H}_{4}\right) ; 3.42(\mathrm{~s}$, $\left.3 \mathrm{H}, \mathrm{OCH}_{3}\right) ; 3.80\left(\mathrm{~s}, 3 \mathrm{H}, \mathrm{Ar}-\mathrm{OCH}_{3}\right) ; 4.44\left(\mathrm{t}, 1 \mathrm{H}, \mathrm{H}_{5}\right)$; $5.14\left(\mathrm{t}, 1 \mathrm{H}, \mathrm{H}_{3}\right) ; 7.28(\mathrm{~d}, 2 \mathrm{H}, \mathrm{Ar}-\mathrm{H}) ; 7.42(\mathrm{~d}, 2 \mathrm{H}, \mathrm{Ar}-\mathrm{H}$, $\mathrm{BP}) ; 7.52$ (t, 1H, Ar-H, BP); $7.63(\mathrm{t}, 2 \mathrm{H}, \mathrm{Ar}-\mathrm{H}, \mathrm{BP})$; $7.68(\mathrm{~d}, 2 \mathrm{H}, \mathrm{Ar}-\mathrm{H}) ; 7.79(\mathrm{~d}, 4 \mathrm{H}, \mathrm{Ar}-\mathrm{H}, \mathrm{BP})$.

IR (Nujol): $v\left(\mathrm{~cm}^{-1}\right)$ : $1726(\mathrm{C}=\mathrm{O}) ; 1708(\mathrm{CO}) ; 1240$ (NO).

\subsubsection{Synthesis of 2-(4-fluorophenyl)-3-biphenyl-5-} cyano isoxazolidines $(\mathbf{3} \boldsymbol{c}(\boldsymbol{i}))$

It was obtained from equimolar mixture of $C$-(4biphenyl)- $N$-(4-fluorophenyl)nitrone (2c) $(500 \mathrm{mg})$ and acrylonitrile $(0.30 \mathrm{~mL})$.

${ }^{1} \mathrm{H}-\mathrm{NMR} \delta(\mathrm{ppm}): 2.83-3.01\left(\mathrm{q}, 2 \mathrm{H}, \mathrm{H}_{4}\right) ; 4.46(\mathrm{t}, 1 \mathrm{H}$, $\left.\mathrm{H}_{5}\right) ; 5.10\left(\mathrm{t}, 1 \mathrm{H}, \mathrm{H}_{3}\right) ; 7.28(\mathrm{~d}, 2 \mathrm{H}, \mathrm{Ar}-\mathrm{H}) ; 7.41(\mathrm{~d}, 2 \mathrm{H}$, $\mathrm{Ar}-\mathrm{H}(\mathrm{BP}) ; 7.52(\mathrm{t}, 1 \mathrm{H}, \mathrm{Ar}-\mathrm{H}(\mathrm{BP}) ; 7.64(\mathrm{t}, 2 \mathrm{H}, \mathrm{Ar}-$ $\mathrm{H}(\mathrm{BP}) ; 7.77$ (d, 2H, Ar-H); 7.69 (d, 4H, Ar-H(BP).

IR (Nujol): $v\left(\mathrm{~cm}^{-1}\right)$ : 1734 (CO); 1274 (NO).

\subsubsection{Synthesis of 2-(4-fluorophenyl)-3-biphenyl-5-} phenyl isoxazolidines $(\mathbf{3} \boldsymbol{c}(\boldsymbol{i i}))$

It was obtained from equimolar mixture of $C$-(4biphenyl)- $N$-(4-fluorophenyl)nitrone (2c) $(500 \mathrm{mg})$ and styrene $(0.25 \mathrm{~mL})$. 
${ }^{1} \mathrm{H}-\mathrm{NMR} \delta(\mathrm{ppm}): 2.88-3.06\left(\mathrm{q}, 2 \mathrm{H}, \mathrm{H}_{4}\right) ; 5.05(\mathrm{t}, 1 \mathrm{H}$, $\left.\mathrm{H}_{5}\right) ; 5.14\left(\mathrm{t}, 1 \mathrm{H}, \mathrm{H}_{3}\right) ; 7.29(\mathrm{~d}, 2 \mathrm{H}, \mathrm{Ar}-\mathrm{H}) ; 7.29-7.41(\mathrm{~m}$, $5 \mathrm{H}, \mathrm{Ar}-\mathrm{H}) ; 7.42(\mathrm{~d}, 2 \mathrm{H}, \mathrm{Ar}-\mathrm{H}(\mathrm{BP}) ; 7.52(\mathrm{t}, 1 \mathrm{H}, \mathrm{Ar}-$ $\mathrm{H}(\mathrm{BP}) ; 7.64$ (t, 2H, $\mathrm{Ar}-\mathrm{H}(\mathrm{BP}) ; 7.77$ (d, 2H, Ar-H); 7.79 (d, $4 \mathrm{H}, \mathrm{Ar}-\mathrm{H}(\mathrm{BP})$.

IR (Nujol): $v\left(\mathrm{~cm}^{-1}\right): 1700$ (CO); 1240 (NO).

\subsubsection{Synthesis of 2-(4-fluorophenyl)-3-biphenyl-5-} benzoate isoxazolidines ( $\mathbf{3} \boldsymbol{c}(\mathbf{i i i})$ )

It was obtained from equimolar mixture of $C$-(4biphenyl)- $N$-(4-fluorophenyl)nitrone (2c) $(500 \mathrm{mg})$ and vinyl benzoate $(0.15 \mathrm{~mL})$.

$\left.{ }^{1} \mathrm{H}-\mathrm{NMR} \delta(\mathrm{ppm})\right): 2.85-3.03\left(\mathrm{q}, 2 \mathrm{H}, \mathrm{H}_{4}\right) ; 4.72(\mathrm{t}$, $\left.1 \mathrm{H}, \mathrm{H}_{5}\right) ; 5.15$ (t, $\left.1 \mathrm{H}, \mathrm{H}_{3}\right) ; 7.28(\mathrm{~d}, 2 \mathrm{H}, \mathrm{Ar}-\mathrm{H}) ; 7.29-7.50$ $(\mathrm{m}, 3 \mathrm{H}, \mathrm{Ar}-\mathrm{H}) ; 7.42$ (d, 2H, Ar-H, BP); $7.53(\mathrm{t}, 1 \mathrm{H}$, $\mathrm{Ar}-\mathrm{H}, \mathrm{BP}) ; 7.64$ (t, 2H, Ar-H, BP); 7.78 (d, 2H, Ar$\mathrm{H}) ; 7.79(\mathrm{~d}, 4 \mathrm{H}, \mathrm{Ar}-\mathrm{H}, \mathrm{BP}) .7 .81(\mathrm{~d}, 2 \mathrm{H}, \mathrm{Ar}-\mathrm{H})$.

IR (Nujol): $v\left(\mathrm{~cm}^{-1}\right): 1738(\mathrm{C}=\mathrm{O}) ; 1730(\mathrm{CO}) ; 1268$ (NO).

\subsubsection{Synthesis of 2-(4-fluorophenyl)-3-biphenyl-5-} ethylate isoxazolidines $(\mathbf{3} \boldsymbol{c}(\boldsymbol{i v}))$

It was obtained from equimolar mixture of $C$-(4biphenyl)- $N$-(4-fluorophenyl)nitrone (2c) $(500 \mathrm{mg})$ and ethyl acrylate $(0.21 \mathrm{~mL})$.

${ }^{1} \mathrm{H}-\mathrm{NMR} \delta(\mathrm{ppm}): 0.93\left(\mathrm{t}, 3 \mathrm{H}, \mathrm{CH}_{3}\right) ; 2.84-3.02(\mathrm{q}$, $\left.2 \mathrm{H}, \mathrm{H}_{4}\right) ; 3.62\left(\mathrm{q}, 2 \mathrm{H}, \mathrm{CH}_{2}\right) ; 4.44\left(\mathrm{t}, 1 \mathrm{H}, \mathrm{H}_{5}\right) ; 5.13(\mathrm{t}, 1 \mathrm{H}$, $\left.\mathrm{H}_{3}\right) ; 7.28(\mathrm{~d}, 2 \mathrm{H}, \mathrm{Ar}-\mathrm{H}) ; 7.41(\mathrm{~d}, 2 \mathrm{H}, \mathrm{Ar}-\mathrm{H}, \mathrm{BP}) ; 7.52$ (t, 1H, Ar-H, BP); 7.64 (t, 2H, Ar-H, BP); 7.77 (d, 2H, $\mathrm{Ar}-\mathrm{H}) ; 7.79$ (d, 4H, Ar-H, BP).

IR (Nujol): $v\left(\mathrm{~cm}^{-1}\right): 1722(\mathrm{C}=\mathrm{O}) ; 1700(\mathrm{CO}) ; 1232$ (NO).

\subsubsection{Synthesis of 2-(4-fluorophenyl)-3-biphenyl-5-} methylene acetate isoxazolidines $(\boldsymbol{3} \boldsymbol{c}(\boldsymbol{v}))$

It was obtained from equimolar mixture of $C$-(4biphenyl)- $N$-(4-fluorophenyl)nitrone (2c) $(500 \mathrm{mg}$ ) and allyl acetate $(0.1 \mathrm{~mL})$.

${ }^{1} \mathrm{H}-\mathrm{NMR} \delta$ (ppm): $2.42\left(\mathrm{~d}, 2 \mathrm{H}, \mathrm{CH}_{2}\right) ; 2.84-3.02$ (q, $\left.2 \mathrm{H}, \mathrm{H}_{4}\right) ; 3.42\left(\mathrm{~s}, 3 \mathrm{H}, \mathrm{OCH}_{3}\right) ; 4.26\left(\mathrm{t}, 1 \mathrm{H}, \mathrm{H}_{5}\right) ; 5.12(\mathrm{t}$, $\left.1 \mathrm{H}, \mathrm{H}_{3}\right) ; 7.26(\mathrm{~d}, 2 \mathrm{H}, \mathrm{Ar}-\mathrm{H}) ; 7.41(\mathrm{~d}, 2 \mathrm{H}, \mathrm{Ar}-\mathrm{H}, \mathrm{BP})$; 7.52 (t, 1H, Ar-H, BP); 7.64 (t, 2H, Ar-H, BP); 7.77 (d, $2 \mathrm{H}, \mathrm{Ar}-\mathrm{H}) ; 7.79$ (d, 4H, Ar-H, BP).

IR (Nujol): $v\left(\mathrm{~cm}^{-1}\right): 1740$ (CO); 1266 (NO).

\subsubsection{Synthesis of 2-(4-fluorophenyl)-3-biphenyl-5-} methylene hydroxy isxazolidine ( $\mathbf{3} \boldsymbol{c}(\boldsymbol{v i}))$

It was obtained from equimolar mixture of $C$-(4biphenyl)- $N$-(4-fluorophenyl)nitrone (2c) $(500 \mathrm{mg})$ and allyl alcohol $(0.15 \mathrm{~mL})$.

${ }^{1} \mathrm{H}-\mathrm{NMR} \delta$ (ppm): 2.74-2.92 (q, 2H, $\left.\mathrm{H}_{4}\right) ; 3.64(\mathrm{~d}$, $\left.2 \mathrm{H}, \mathrm{CH}_{2}\right) ; 4.76\left(\mathrm{t}, 1 \mathrm{H}, \mathrm{H}_{5}\right) ; 5.12\left(\mathrm{t}, 1 \mathrm{H}, \mathrm{H}_{3}\right) ; 5.25(\mathrm{~s}, 1 \mathrm{H}$, $\mathrm{OH}) ; 7.28(\mathrm{~d}, 2 \mathrm{H}, \mathrm{Ar}-\mathrm{H}) ; 7.41$ (d, 2H, Ar-H, BP); 7.52 (t, 1H, Ar-H, BP); 7.64 (t, 2H, Ar-H, BP); 7.77 (d, 2H, $\mathrm{Ar}-\mathrm{H}) ; 7.79$ (d, 4H, Ar-H, BP).

IR (Nujol): $v\left(\mathrm{~cm}^{-1}\right)$ : $1720(\mathrm{CO}) ; 1248(\mathrm{NO})$.
5.1.26. Synthesis of 2-(4-fluorophenyl)-3-biphenyl-5-(3cyclohexene) isoxazolidines (3c(vii))

It was obtained from equimolar mixture of $C$-(4biphenyl)- $N$-(4-fluorophenyl)nitrone (2c) $(500 \mathrm{mg}$ ) and 4-vinyl-1-cyclohexene $(0.25 \mathrm{~mL})$.

${ }^{1} \mathrm{H}-\mathrm{NMR} \delta(\mathrm{ppm}): 1.38-1.42\left(\mathrm{~m}, 2 \mathrm{H}, \mathrm{CH}_{2}\right.$, cyclohexene); $1.52(\mathrm{~s}, 1 \mathrm{H}$, cyclohexene); $2.28-2.31(\mathrm{~m}, 2 \mathrm{H}$, cyclohexene); $2.33-2.38$ (m, 2H, cyclohexene); 2.85$3.03\left(\mathrm{q}, 2 \mathrm{H}, \mathrm{H}_{4}\right) ; 4.82(\mathrm{~m}, 1 \mathrm{H}, \mathrm{CH}) ; 5.14\left(\mathrm{t}, 1 \mathrm{H}, \mathrm{H}_{3}\right)$; 5.6-5.66 (m, 2H, cyclohexene); $7.28(\mathrm{~d}, 2 \mathrm{H}, \mathrm{Ar}-\mathrm{H}) ; 7.41$ (d, 2H, Ar-H, BP); $7.52(\mathrm{t}, 1 \mathrm{H}, \mathrm{Ar}-\mathrm{H}, \mathrm{BP}) ; 7.63(\mathrm{t}, 2 \mathrm{H}$, $\mathrm{Ar}-\mathrm{H}, \mathrm{BP}) ; 7.77(\mathrm{~d}, 2 \mathrm{H}, \mathrm{Ar}-\mathrm{H}) ; 7.79(\mathrm{~d}, 4 \mathrm{H}, \mathrm{Ar}-\mathrm{H}$, $\mathrm{BP})$.

IR (Nujol): $v\left(\mathrm{~cm}^{-1}\right)$ : $1730(\mathrm{CO}) ; 1260$ (NO).

\subsubsection{Synthesis of 2-(4-fluorophenyl)-3-biphenyl-5-} ethyl isoxazolidines (3c(viii))

It was obtained from equimolar mixture of $C$-(4biphenyl)- $N$-(4-fluorophenyl)nitrone (2c) $(500 \mathrm{mg})$ and methyl acrylate $(0.35 \mathrm{~mL})$.

${ }^{1} \mathrm{H}-\mathrm{NMR} \delta$ (ppm): $2.87-3.05$ (q, 2H, $\left.\mathrm{H}_{4}\right) ; 3.41(\mathrm{~s}, 3 \mathrm{H}$, $\left.\mathrm{OCH}_{3}\right) ; 4.45\left(\mathrm{t}, 1 \mathrm{H}, \mathrm{H}_{5}\right) ; 5.12\left(\mathrm{t}, 1 \mathrm{H}, \mathrm{H}_{3}\right) ; 7.28(\mathrm{~d}, 2 \mathrm{H}$, $\mathrm{Ar}-\mathrm{H}) ; 7.41(\mathrm{~d}, 2 \mathrm{H}, \mathrm{Ar}-\mathrm{H}, \mathrm{BP}) ; 7.52(\mathrm{t}, 1 \mathrm{H}, \mathrm{Ar}-\mathrm{H}$, BP); 7.64 (t, 2H, Ar-H, BP); 7.77 (d, 2H, Ar-H); 7.79 (d, $4 \mathrm{H}, \mathrm{Ar}-\mathrm{H}, \mathrm{BP})$.

IR (Nujol): $v\left(\mathrm{~cm}^{-1}\right)$ : 1745 (CO); 1278 (NO).

\subsection{Determination of antifungal activity}

Fungus used for the antifungal activity: Aspergillus flavus (ATCC76087) Fusarium moniliforme (ATCC5342) Botrydiplodia theobromae (ATCC76087)

The isoxazolidines were tested for antifungal activity by serial tube dilution technique $[13,14]$ at different concentrations $(0.5,1,1.5, \ldots, 12 \mathrm{mM})$ against $A$. flavus, $F$. moniliforme and $B$. theobromae. Nystatin was used as reference standard and $\mathrm{CHCl}_{3}$ as control. To the culture tubes containing $1.9 \mathrm{~mL}$ of media, $0.1 \mathrm{~mL}$ of test solution was added at sterile conditions. To all the tubes including standard and controls, the fresh inoculum was added using Himedia flexiloop 4 calibrated to $0.001 \mathrm{~mL}$. After incubating all the tubes at $37^{\circ} \mathrm{C}$ for $24 \mathrm{~h}$, their absorbance was recorded at $640 \mathrm{~nm}$ along with Nystatin. Percentage of inhibition was calculated as by the following equation,

$\%$ Inhibition $=\frac{100(P-Q)}{P}$

where $P=$ absorbance without the test sample and $Q=$ absorbance with test sample.

\section{References}

[1] R.C. Heel, R.N. Brogden, A. Carmine, P.A. Morley, T.M. Speight, G.S. Avery, Drugs 23 (1982) 1-5. 
[2] K.R. Ravikumar, H. Mallesha, K.S. Rangappa, Synthetic communication (Accepted SC-2002-128).

[3] K.R. Ravikumar, H. Mallesha, K.S. Rangappa Archiv der Phrmazie (Accepted FP 701).

[4] H. Mallesh, K.R. Ravikumar, B.K. Vishukumar, K. Mantelingu, K.S. Rangappa, Proc. Indian Acad. Sci. (Chem. Sci.) 1113 (4) (2001) 291-296.

[5] J.S. Splitter, M. Calvin, J. Org. Chem. 23 (1958) 651-655.

[6] M.J. Kamlet, L.A. Kaplan, J. Org. Chem. 22 (1957) 576-579.

[7] C.D. Black, St., R.F. Crozier, V.C. Davis, Synthesis 4 (1975) 205-221.

[8] A. Banerji, A.J. Sahu, Scient. Indus. Res. 45 (1986) 355 369.
[9] H. Mallesha, K.R. Ravikumar, K. Mantelingu, K.S. Rangappa, Synthesis 10 (2001) 1459-1461

[10] H. Mallesha, K.R. Ravikumar, K.S. Rangappa, Synthesis 16 (2001) 2415-2418.

[11] H. Mallesha, K.R. Ravikumar, K.S. Rangappa, Nucleosides Nucleotides Nucleic acids 21 (4\&5) (2002) 385-392.

[12] K.B.G. Torsell, Nitrile Oxides, Nitrone and Nitronates in Organic Synthesis, VCH, New York, Weinheim, 1988.

[13] C.H. Collins, P.M. Lyne, Microb. Methods, Butterworth, London, 1970.

[14] B.G. Mullen, R.T. Decory, T.J. Mitchell, D.S. Allen, C.R. Kinsolving Vassil St. Georgiev. J. Med. Chem. 31 (1988) $2008-$ 2014. 